\title{
The Research on Physical Distribution Trainer Control System
}

\author{
Shumei Liu \\ Shandong University of Science and Technology \\ Qingdao, Shandong, China \\ 707408310@qq.com \\ Guanguo Ma \\ Shandong University of Science and Technology \\ Qingdao, Shandong, China \\ 156154682@qq.com
}

\author{
Kailin Feng \\ Shandong University of Science and Technology \\ Qingdao, Shandong, China \\ 767416732@qq.com
}

\begin{abstract}
Based on the law of athlete physical distribution in this paper, an athlete physical distribution trainer is designed and studied. It can help athletes in track class sports rational allocation of physical training to improve sports performance. This article describes the working principle of physical distribution trainer, explores the trajectory guide on the spot and the function relationship between speed and motor rotation on this basis, designs composition of the physical distribution control system of training, and builds the basis of the control PID control algorithm system, which provides a theoretical basis of control systems for the design and study of physical distribution trainer.
\end{abstract}

Keywords-Physical Distribution Trainer; Physical Distribution; Functional Relationship; Control System; PID Control Algorithm)

\section{INTRODUCTION}

In order to achieve better results in the 1500 meters speed skating and 5,000 meters long-distance running long distance sports, athletes need not only better physical fitness, motor skills and the necessary relevant will, but also enough scientific and rational allocation of physical strength to ensure the strength of the full. Because of the limited overall force of people, in order to give full play to the rational allocation of physical strength during the race, athletes should not have inadequate post-physical. Therefore, in peacetime training, athletes need to strengthen exercises of the physical distribution, and the athletes themselves have obviously inaccurate intuitive feeling, so they need the appropriate instrument for the guidance of training. A design capable of directing physical distribution athlete trainer is studied in this paper, which is able to help athletes enhance this training better. It uses the runway in the middle square of light projected onto the track in accordance with the design speed of the movement of colored light points to lead the reasonable physical distribution for athletes, in order to achieve better results in the race.
II. WORKING PRINCIPLE OF PHYSICAL DISTRIBUTION TRAINER

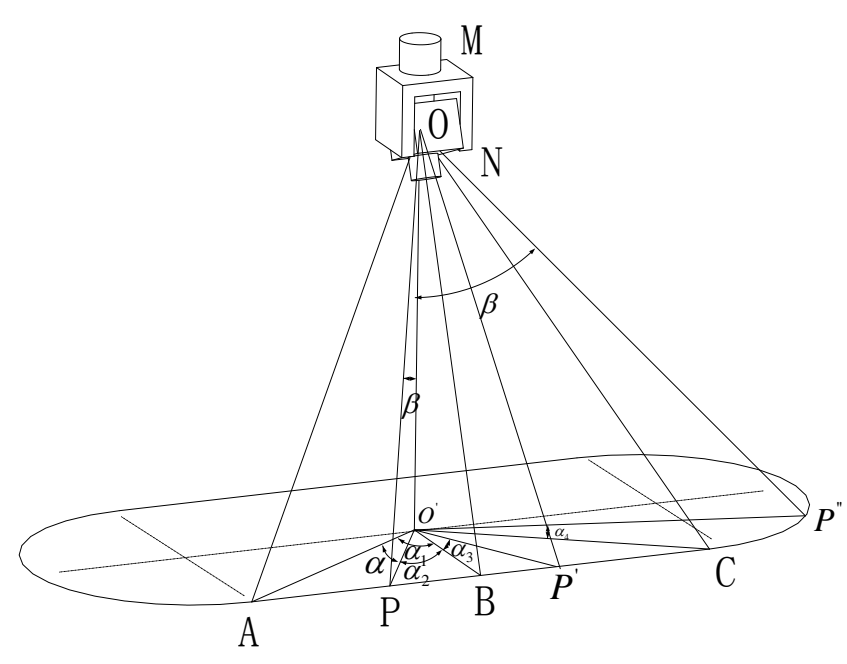

Figure 1. Working diagram of physical distribution trainer

The working principle of the physical distribution trainer can be described as Fig. 1. The light source is installed on the top of the gymnasium center, and the light source driven by motor $\mathrm{M}$ and motor $\mathrm{N}$ can be rotated around the upright axle and swinged around the horizontal axle. The colored light spot that this light source shoots out and projected on the runway can runs according to the setting track and speed, by the accurate control of these two motors. Researchers just use the colored spot that can change the track and speed to guide the athletes to exercise. In this way, athletes can exercise with a science and reasonable speed distribution, in which athletes can run with different speed at different part. Therefore, the result of athlete strength can be distributed reasonablely. 


\section{RELATIONS OF SPOT MOVEMENT AND MOTOR ROTATION}

For irradiated spot on the ground can be completed in accordance with the route set from straight to arc, then uniform motion from arc to straight line, the need to rotate the motor and the motor can be achieved with a pitch

As shown in Fig. 1, in line segment $\mathrm{AB}$ and start half of the curve part as an example, the spot movement and rotation of the two stand-alone relationship (straight line segment BC and the relationship between the arc segment in this author on the paper, "the design of athlete's physical distribution training" in detail). Uniform motion speed $\mathrm{v}$ is set, it is possible to set the speed $\mathrm{v}$ is a constant; the size of the set speed can be adjusted by remote control.

When the light spot movement on a straight line segment $\mathrm{AB}$, at this time $0 \leq v t<l / 2$.

$$
\begin{gathered}
\alpha=\angle A O^{\prime} P=\alpha_{1}-\alpha_{2}=\alpha_{1}-\arctan \frac{l / 2-v t}{R} \\
\beta=\angle P O O^{\prime}=\arctan \frac{O^{\prime} P}{O O^{\prime}}=\arctan \frac{\sqrt{(l / 2-v t)^{2}+R^{2}}}{h}
\end{gathered}
$$

In these equations:

$\mathrm{P}$ is any point on the line segment light motion point, then $A P=v t, \quad B P=l / 2-v t$;

$l \_$Runway length line segment

$\mathrm{R}$ - The radius of the arc segment

$h$ - Light height from the ground

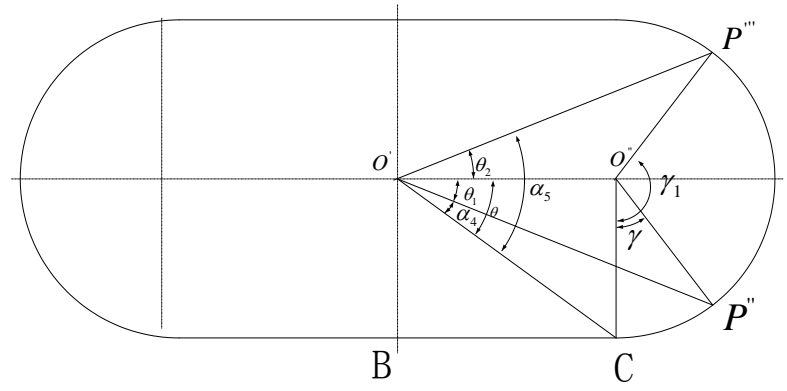

Figure 2. The geometric sketch of the track plane

When the guiding spot is at the curve part, the spot can be showed as Fig. 2. At this time: $0<\gamma<\pi / 2$

$O^{\prime} P^{\prime \prime}=\sqrt{(l / 2)^{2}+R^{2}-2 \times(l / 2) \times R \times \cos (\pi / 2+\gamma)}$

$=\sqrt{l^{2} / 4+\mathrm{R}^{2}+l R \sin \gamma}$

$\theta_{1}=\arccos \left[\frac{\left(O^{\prime} O^{\prime \prime}\right)^{2}+\left(O^{\prime} P^{\prime \prime}\right)^{2}-\left(O^{\prime} P^{\prime \prime}\right)^{2}}{2\left(O^{\prime} O^{\prime \prime}\right) \times\left(O^{\prime} P^{\prime \prime}\right)}\right]$

$=\arccos \left(\frac{l^{2}+2 l R \cos \gamma}{2 l \times \sqrt{l^{2} / 4+\mathrm{R}^{2}+l R \sin \gamma}}\right)$

$\alpha=\angle A O^{\prime} P^{\prime \prime}=2 \alpha_{1}+\alpha_{4}$

$=2 \alpha_{1}+\arctan \frac{2 R}{l}-\arccos \left(\frac{l^{2}+2 l R \cos \gamma}{2 l \times \sqrt{l^{2} / 4+\mathrm{R}^{2}+l R \sin \gamma}}\right)$

$$
\begin{aligned}
& \beta=\angle P^{\prime \prime} O O^{\prime}=\arctan \frac{O^{\prime} P^{\prime \prime}}{O O^{\prime}} \\
& =\arctan \frac{\sqrt{l^{2} / 4+\mathrm{R}^{2}+l R \sin \gamma}}{h}
\end{aligned}
$$

In this equation:

$$
\angle O^{\prime} O^{\prime \prime} P^{\prime \prime}=\pi / 2+\gamma=\pi / 2+\frac{v t-l}{R}
$$

\section{THE DESIGNAL OF CONTROL SYSTEM ARCHITECTURE}

The whole construction of control system of the physical distribution trainer can be described as Fig. 3. The main system of the trainer conclude a CUP that researchers use SCM of STM32 to complete, a rotating motor, a swing motor, an encoder, a data acquisition module, a set of remote control module and so on. When the remote give the signal that orders the motors to open out, the rotating motor and the swing motor start to work. And then the encoders installed on these two motors start to detect the angel these two motors rotate, and put the signal out. Then the data acquisition module collects this signal, and puts it into the CPU. The CPU gives the control signal to control the speed of these two motors, according to the principle of the spot and the speed. And the CUP responses and compares them, after which the system of the physical distribution trainer can be in a closed-loop control. At the same time, the speed of the spot to guide the athlete can be showed on the screen of the remote by the remote control module.

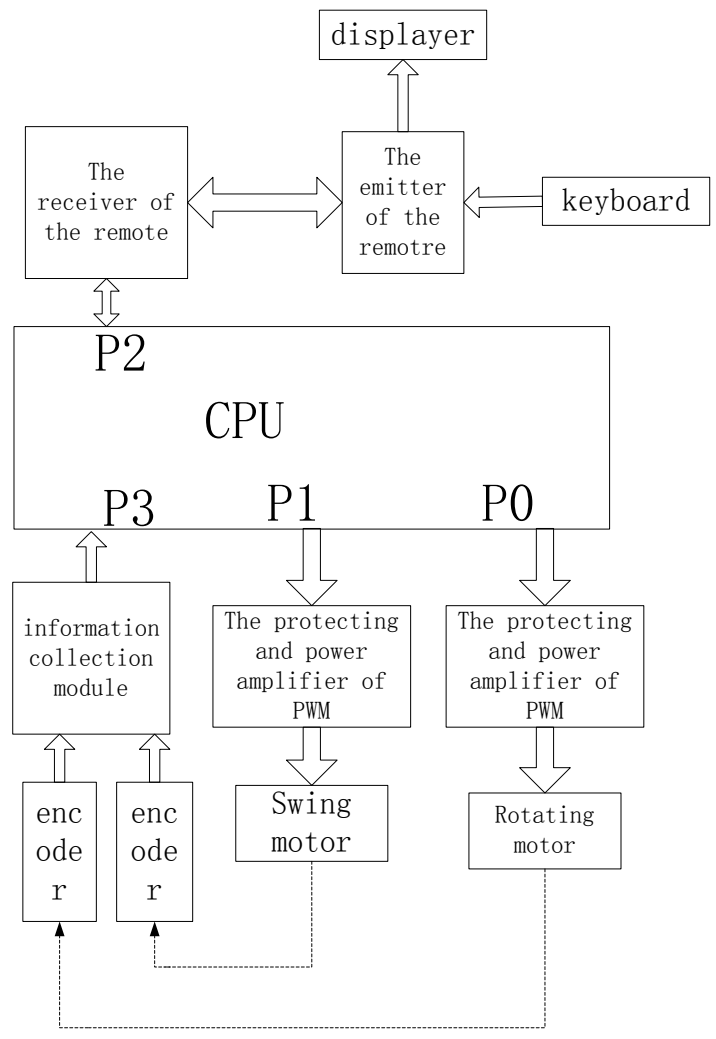

Figure 3. The control block of Athletes trainer physical distribution 
When Athletes need to change the speed, they can adjust the keyboard on the remote control through the re mote control receiver will signal to the central proces sor, the central processing unit to re-adjust the motor speed.

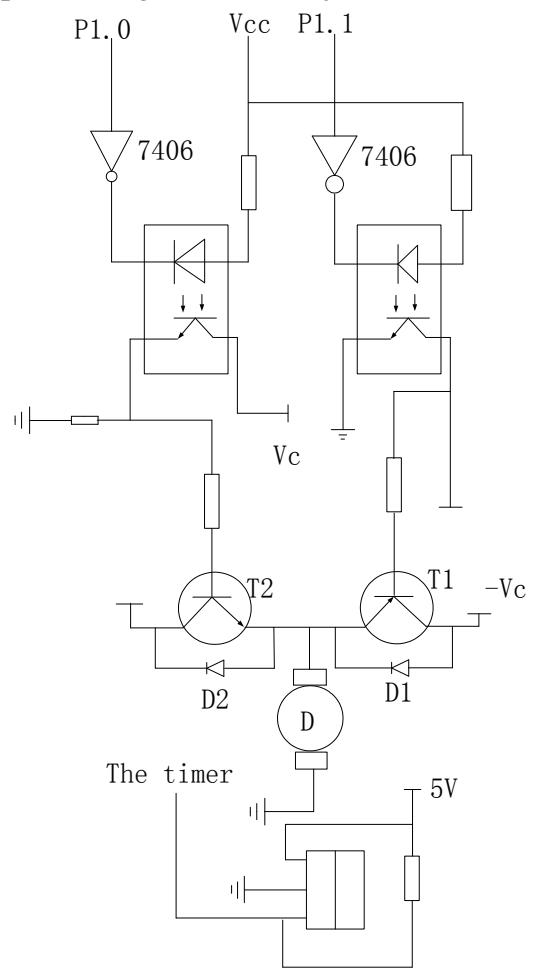

Figure 4. Drive and main loop control circuit

The control system of the physical distribution trainer drive module and the main circuit can be showed as Fig. 4. The control program of the control system, the parameter of PID and the setting speed can be put into the CPU before the equipment being installed. When the physical distribution trainer starts to work, the signal, including starting, stopping and reset, was put in by the keyboard on the remote emitter. The control signal is produced by speed closed-link system and PID operation on the SCM of STM32. Besides, the control signal was transformed into PWM signal that have different duty cycle. Also, the PWM signal was put out through the corresponding endpoint. Then the PWM signal come into the motor driver, which is called protesting and magnifying PWM signal module. Then the electricity input the motors to drive them to rotate.

When the athletes start to train and the command of starting was given out or when the reset command was given out and the guiding spot is at the end half of the track, the motors need to rotate with the forward direction. At this moment, port 1.0 puts the low level out and the high level is put out at the port 1.1. Meanwhile the switching triode $\mathrm{T} 1$ is conductive, which can make the motor armature between the forward voltages. Then the motor rotates with the forward direction. On the contrary, when the guiding spot is at the top half of the track, and the command of reset is put out, researchers need the motors to rotate with reverse direction. At the moment, the port 1.0 put the high level out and the port 1.1 put the low level out. Meanwhile the switching triode T2 is conductive, which can make the motor armature between the reverse voltages. Then the motors need to rotate with the reverse direction.

In this control system, DC motors is used, and the incremental encoder is used as the speed detection module, which can transform the electric signal that was produced by motors to count impulse. And the angel of the motors can be showed by the number of the count impulse produced by encoders.

\section{THE DESIGN OF CONTROL SYSTEM SOFT WARE}

The physical distribution control system of the main trainer module main program, T0 interrupt subroutine, INT0 interrupt subroutine, PID algorithm subroutine, remote module embedded in the program and keyboard display subroutine. After a series of initialization the main program into the PWM drive routines waits interrupt. External interrupt routine INT0 count the data of Motor speed pulse which is input by acquisition module. T0 interrupt service routine count the number of interrupts. Readout speed pulse counts the value every $1 \mathrm{~s}$. The speed is calculated. Meanwhile PID subroutine is called to compare test speed and set speed. Control instructions drawn into the PWM controller to adjust the two motors.

In the design of actual closed-loop control system, PID controller is a frequently-used control way. The speed of the motors is approximately proportional to the voltage out of armature, which is the control thinking of PID. When the signal is collected, researchers can catch a relation described as following:

$$
t=i \times T
$$

In this equation:

$T$-sampling period;

$i$-positive integer.

The law of simulating the PID can be described by differential equation as following:

$$
u(t)=K_{p}\left[e(t)+\frac{1}{T_{i}} \int e(t) d t+T_{d} \bullet \frac{d e(t)}{d t}\right]
$$

In this equation:

$u(t)$ - the outputting of the regulator at the time of ' $\mathrm{t}$ '.

$K_{p}$-proportionality coefficient.

$e(t)$ - the input value of regulator ( it is general deviation value)

$T_{i}$-integral time constant.

$T_{d}$ - differentiating time constant.

In the progress of the physical distribution trainer working, the CPU can only recognize the digital quantity, instead of continuous control equation, so continuous analog signal cannot be recognized and calculated by the CUP. That is why researchers must dispose the control law by discretizing arithmetic design. The observation and control system of motor speed is discrete type control system about time, so the digital difference equation can be used to replace the differential equation in continuous control system. And in this way, the PID equation can be discretized.

The difference equation of the control system can be described as following: 


$$
u_{(i)}=u_{(i-1)}+K_{p}\left(e_{i}-e_{i-1}\right)+K_{p} \frac{T}{T_{i}} e_{i}+K_{p} \frac{T_{d}}{T}\left(e_{i}-2 e_{i-1}-e_{i-2}\right)
$$

If researchers change the parameters as following

$$
\begin{aligned}
& P=K_{p} \\
& I=K_{p} \times T / T_{i} \\
& D=K_{p} \times T_{d} / T
\end{aligned}
$$

According to PID algorithm design ideas and discrete PID equation, obtain its differential equation:

$u_{(i)}=u_{(i-1)}+P\left(e_{i}-e_{i-1}\right)+I e_{i}+D\left(e_{i}-2 e_{i-1}-e_{i-2}\right)$

Wherein, $P 、 I 、 D$ are the proportional gain, integral coefficient and differential coefficient.

According to Equation 3 and the specific circumstances of this physical distribution trainer, design a PID algorithm subroutine shown in Fig. 5

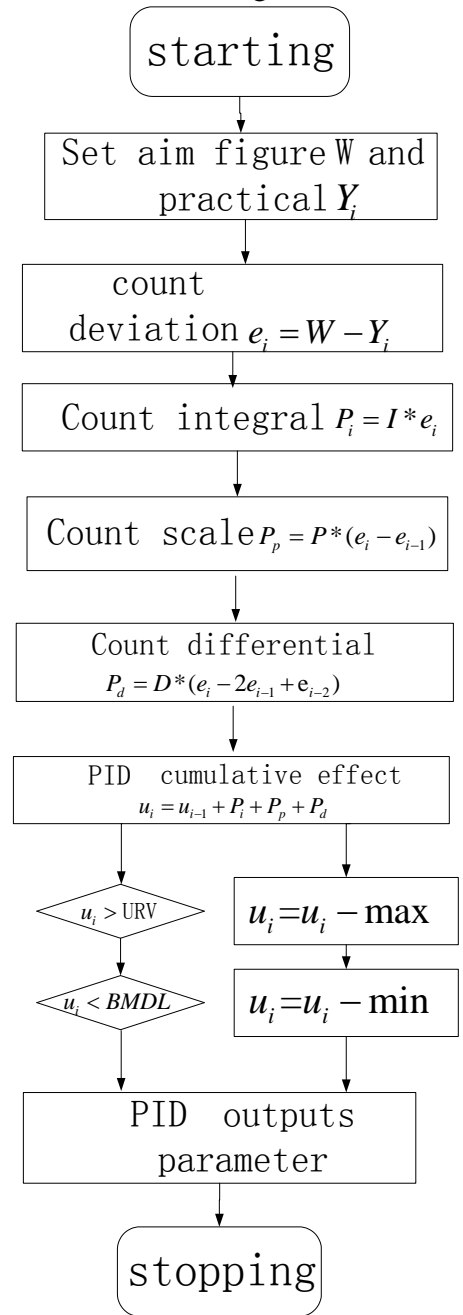

Figure 5. PID algorithm module subprogram

During operation of the system, through STM32 internal timer control, interrupt every $\mathrm{T} s$, complete a PID control calculations, and constantly adjust the controlled parameter. The main program of the PWM drive module constantly adjusts the output value adjustment PWM waveform control parameters based on comparison, to achieve the real-time control motor speed task. In the program provides an upper limit output size $u_{i}-\max$ and the lower limit output size.

It can prevent system crashes on motor control in emergency situation.

\section{CONCLUSIONS}

A kind of physical strength distribution trainer was introduced in the article. The light source is drived to change its posture by the cooperating movement of rotating motor and swing motor, which can make the colored spot shoot the runway runs according to the specified track and speed. In this way, the physical strength distribution trainer can play a part of guiding athletes exercise with a reasonable strength. And the working principle of the physical strength distribution trainer is described in this article. It explores the spot velocity and trajectory with the motor rotation has a certain function. And based on the theory, the whole structure of the control system is explored, and the PID arithmetic based on this control system is also researched. The control system is perfectly adapted to the physical distribution of such training devices, both to ensure that the design requirements, but also to reduce system failure occurs

\section{REFERENCES}

[1] Yu Baihui. The introduction of the distributing strength in middlelong-dist ancerace[J]. Exchange of experience, 2014(6),120-121.

[2] Pu lianggui, Ji Minggang. Design of Mechinery[M]. Beijing, Higher Education Press, 2003.

[3] Cheng Daxian. Hand Book of Mechanical Design[K].Beijing, Chemical Industry Press, 2004.

[4] Wang Junfeng, Xue Hongde. Modern control technology and application[M].Beijing: Posts \& Telecom Press, 2005.

[5] Li Tiantian, Chen Hong, Li Jingtao. Feedback Control of DC Mot or by PID Algorithm[J]. Electro-Optic Technology Application, 2009(12),55-57.

[6] Sun Yuanwen. The DC motor control based on the MCU[J]. Science \&Technology information, 2010(35),1154-1155.

[7] Zhu Lisheng. A survey on DC motor closed- loop control technology[D]. Nanjng: Nanjing University of Science and Technology, 2009.

[8] Du Yingcai, Wang Xijun, Wang Shujie, and Therefore, on. Autodetection system of incremental encoder[J]. Journal of Electronic Measurement and Instrument, 2012(11),993-997.

[9] Bao Song, Bao Kejin, Yu Jinghua. Measurement and Control System of Direct Current Electromotor Based on PID and SCM[J]. Microcomputer Development, 2003(8), 72-74.

[10] Tong Qiangmin. A sort of Wireless Romote Control Technology Based SCM System[J]. Computer Knowledge and Technology, 2009(3), 2021-2022.

[11] Zhang Hongt ao, Yan Lin, Xu Xiaohui, and Therefore, on. Research on Brushless DC Mot or Control System Based on PID and SCM[J]. Modern Electronic Technology, 2007(10),52-54.

Corresponding author: Feng Kai-lin; TEL:13969849679; Email:156154682@qq.com. 